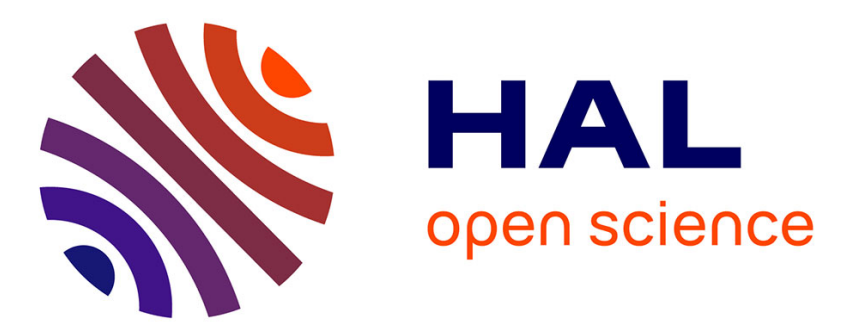

\title{
A thermodynamic approach to effective stresses in unsaturated soils incorporating the concept of partial pore deformations
}

Patrick Dangla, Jean-Michel Pereira

\section{- To cite this version:}

Patrick Dangla, Jean-Michel Pereira. A thermodynamic approach to effective stresses in unsaturated soils incorporating the concept of partial pore deformations. Vadose Zone Journal, 2014, pp.2136-2136. 10.2136/vzj2013.06.0110 . hal-01157350

\section{HAL Id: hal-01157350 \\ https://hal-enpc.archives-ouvertes.fr/hal-01157350}

Submitted on 8 May 2019

HAL is a multi-disciplinary open access archive for the deposit and dissemination of scientific research documents, whether they are published or not. The documents may come from teaching and research institutions in France or abroad, or from public or private research centers.
L'archive ouverte pluridisciplinaire HAL, est destinée au dépôt et à la diffusion de documents scientifiques de niveau recherche, publiés ou non, émanant des établissements d'enseignement et de recherche français ou étrangers, des laboratoires publics ou privés. 
1. A thermodynamic approach to effective stresses in unsaturated soils incorporating the concept of partial pore deformations

6 IFSTTAR, F-77420 Marne-la-Vallée

$7 \quad *$ : Corresponding author Abstract
The thermodynamical analysis presented here follows from the work
of Coussy et al [13] who proposed a thermodynamically consistent model
for unsaturated soils which is based on a Bishop-like effective stress to
describe the stress-strain relationship while the water saturation (or the
capillary pressure) is involved in a saturation-induced hardening in ad-
dition to the mechanical hardening. We extended this model to include
the effect of interfaces in the mechanical behaviour and we showed that
the Bishop-like stresses involved in the elastic and plastic responses re-
spectively can take different expressions. The Modified Cam-Clay model
used for saturated soils is extended to unsaturated soils through the use of 

results reported from the literature.

\section{${ }_{22} 1$ Introduction}

${ }_{23}$ The concept of effective stress in unsaturated soils goes back to the work of

${ }_{24}$ Bishop [4] who extended the concept of Terzaghi's effective stress by introducing

25 a weighted average of gas and liquid pressures [5, 7]. This proposal encountered

26 difficulties in explaining collapse behaviour $[6,9,1,23]$. Then many authors

${ }_{27}$ have pointed out the need of two independent stress state variables to account

28 for experimental observations on the mechanical behavior of unsaturated soils

29 [18]. On that basis elastoplastic models were formulated $[2,20]$. These models

30 can be viewed as an extension of the Cam-Clay model to unsaturated situations.

31 This has launched the development of many other models $[24,30,7,28,29,19$,

$3236,34,15,33]$. All these models are founded on two independent stresses even

33 though they vary widely in the choice of the stresses. Some of them [32] chose to

34 refer one stress to a Bishop-type stress. But all those models require the suction

35 or the capillary pressure as an additional and independent stress. The reader

36 can refer to the comprehensive review of effective stresses proposed by Nuth et

37 al [31]. In most of these models suction is a hardening variable and thus has a

38 status somehow different from the Bishop stress. As noted by Coussy [13, 12]

39 the status of the suction or capillary pressure is two fold. Its variations control 
the fluid invasion process through the retention curve and they also control the

${ }_{41}$ mechanical behaviour through the deformation of the pores they induced. This

42 can be a source of confusion in the formulation of the constitutive equations

${ }_{43}$ as pointed out by Alonso et al [3]. A significant breakthrough in the way of

44 clarification, was achieved by Coussy [12] who proposed a more appropriate

45 definition of the saturation degree, called Lagrangian saturation degree. This

46 new definition is only associated to the invasion process, i.e to the creation

${ }_{47}$ and destruction of fluid-solid interface areas during wetting-drying processes.

${ }_{48}$ In contrast this saturation degree is not affected by the deformation process of

49 the porous network. Thanks to this new concept Coussy et al [13] have given

50 a physical background to the coefficient involved in the Bishop effective stress

${ }_{51}$ and proposed, on this physical basis, an extension of the Cam-Clay model to

${ }_{52}$ unsaturated conditions which is thermodynamically consistent. Experimental

${ }_{53}$ data on shear strength suggest that this Bishop coefficient is mostly smaller than

${ }_{54}$ the saturation degree generally used in the expression of the Bishop effective

${ }_{55}$ stress [3].

56 Following the approach of Coussy, we explore here the effect of the interface

57 energy on the mechanical behaviour of unsaturated soils which was neglected

${ }_{58}$ in the work of Coussy $[11,13]$. As opposed to what was done in Coussy, the

${ }_{59}$ interface energy here depends on the deformation of the material. We also derive

60 two Bishop-like effective stresses related to the elastic and plastic responses

${ }_{61}$ respectively. Finally we propose a simple extension of the Modified Cam-Clay

62 model to unsaturated conditions and some comparisons with experimental data 
are shown.

An unsaturated soil consists in a solid skeleton composed of solid grains in contact, a gas phase and a liquid phase. These three phases interact with each other through interfaces which sustain surface stress and possess their own en-

67 ergy. These interfaces play a fondamental role in the thermodynamic analysis of

${ }_{68}$ unsaturated soils. Accordingly the thermodynamics of a representative volume

69 element of unsaturated soil can be addressed by considering three different sys-

70 tems. The first one is the soil itself, as depicted above, including all the matter

${ }_{71}$ in all form contained in the RVE. It is an open thermodynamic system exchang-

72 ing gas and liquid mass. The second system is obtained by removing the bulk

73 fluid masses whatever the fluid form. It is then formed of the solid phase and

74 the interfaces only. This system is still subjected to the gas and liquid pressures

75 through the interfaces. However these pressure are considered now as external

76 forces. Like Coussy [13] we'll call this system the "apparent solid skeleton"

77 (subscript "ske") since it includes interfaces with energy concentrated on those

78 surfaces. By removing the interfaces we can obtain a third system consisting

79 in only the solid phase. We will call it the "solid matrix" (subscript "sol") in

so the following. This system is now subjected to external forces which differ from

${ }_{81}$ the gas and liquid pressures since part of these pressures are absorbed by the

${ }_{82}$ interface surface stresses. We will assume that these forces can be represented

83 by two effective pressures exerted on the part of the solid wall in contact with

${ }_{84}$ the solid-liquid and solid-gas interfaces. We will denote them by $\pi_{\mathrm{L}}$ and $\pi_{\mathrm{G}}$.

${ }_{85}$ We must note that such effective pressures have already been derived formally 
86 by other authors from a microscopical approach and by making use of averaging

87 technics $[21,22]$.

\section{${ }_{88} 2$ Effective pore pressures and interface energy}

89 Consider a volume $V_{0}$ of soil in its undeformed reference configuration. In the

90 current configuration the volume is $V$, the pore volume is $\phi V_{0}$ where $\phi$ is the

${ }_{91}$ Lagrangian porosity [11]. The pore volume occupied by the liquid and gas phase

92 are given by $\phi_{\mathrm{L}} V_{0}$ and $\phi_{\mathrm{G}} V_{0}$, where the $\phi_{\mathrm{J}}(\mathrm{J}=\mathrm{L}, \mathrm{G})$ can be coined as partial

93 Lagrangian porosities respectively. Furthermore we have $\phi_{\mathrm{L}}+\phi_{\mathrm{G}}=\phi$.

${ }_{94}$ The balance free energy of the apparent solid skeleton, at constant temper-

95 ature, can be expressed as [13]

$$
d F_{\text {ske }}=\sigma_{i j} d \epsilon_{i j}+p_{\mathrm{L}} d \phi_{\mathrm{L}}+p_{\mathrm{G}} d \phi_{\mathrm{G}}
$$

${ }_{96}$ The current partial porosity $\phi_{\mathrm{J}}$ can be written in the form [12]

$$
\phi_{\mathrm{J}}=\phi_{0} S_{\mathrm{J}}+\varphi_{\mathrm{J}} ; \quad S_{\mathrm{L}}+S_{\mathrm{G}}=1
$$

${ }_{97}$ where $S_{\mathrm{J}}$ is the Lagrangian saturation degree and $\varphi_{\mathrm{J}}$ is the deformation of the

${ }_{98}$ porous network occupied by the phase $\mathrm{J}$ which can be coined as the partial

99 pore deformation. In Eq. (2) $\phi_{0} S_{\mathrm{J}}$ is the volume occupied by the fluid J prior to any deformation i.e. by the part of the porous volume of the undeformed

101 reference configuration which is delimited by the internal solid walls wetted by 102 the fluid $\mathrm{J}$ [12]. The variations of $S_{\mathrm{J}}$ is therefore associated to the invasion 103 process of interfaces i.e. to the displacement of the common line between the 
122 [13]: "In the familiar capillary case, although the suction can be defined as 123 the difference between the pressures of the non-wetting and wetting phases, the

where $F_{\text {sol }}$ is the free energy stored in the solid matrix and $F_{\text {int }}$ is the free energy of interfaces. The free energy of the solid matrix, $F_{\text {sol }}$, is mainly a function of the deformation variables $\epsilon_{i j}, \varphi_{\mathrm{L}}, \varphi_{\mathrm{G}}$ with $S_{\mathrm{L}}$ intervening as a coupling term. In this sense the derivative $\frac{\partial F_{\text {sol }}}{\partial S_{\mathrm{L}}}$ will always be coupled with the deformation variables and therefore will be considered as a small term compared to $\frac{\partial F_{\text {int }}}{\partial S_{\mathrm{L}}}$. Similarly $F_{\text {int }}$ depends essentially on $S_{\mathrm{L}}$ and the partial deformation of pores, $\varphi_{\mathrm{L}}$ and $\varphi_{\mathrm{G}}$, as coupling terms. Because interfaces are located in the porosity, $F_{\text {int }}$ is assumed as independent of the skeleton strains.

According to Eq. (3) the force-like vector formed by the stress tensor, the fluid pressures and the pressure difference $-\phi_{0}\left(p_{\mathrm{G}}-p_{\mathrm{L}}\right)$ is energy conjugate to the deformation-like vector formed by the strain tensor, the partial pore deformations and the saturation degree. As already noted by Coussy et al (2) 
various role of the pressure difference must be well separated from that of the

${ }_{125}$ suction". Indeed the status of fluid pressures in the energy change is two fold.

126 On one hand the mechanical pressures that are exerted on complementary parts of the solid wall from the liquid and gas, govern the process of deformation of the material. The saturation degree which controls the partition of these pressures on the solid wall can be considered as an independent parameter of the behaviour and therefore decoupled from these mechanical pressures. On the other hand the process of invasion, linked to the change of saturation degree, is controlled by the suction through the retention curve. Even though the suction is eventually given by the difference between the gas and liquid pressures, the status of the suction is here well separated from that of the mechanical pressures.

From a thermodynamical point of view these different status of fluid pres136 sures form three independant forces which are energy conjugate to the three ${ }_{137}$ independent thermodynamical variables: $\varphi_{\mathrm{L}}, \varphi_{\mathrm{G}}, S_{\mathrm{L}}$.

\subsection{Energy of the solid matrix}

139 Combining (3) and (4), the free energy of the sole solid matrix satisfies

$$
\left(d F_{\mathrm{sol}}\right)_{S_{\mathrm{L}}}=\sigma_{i j} d \epsilon_{i j}+\pi_{\mathrm{L}} d \varphi_{\mathrm{L}}+\pi_{\mathrm{G}} d \varphi_{\mathrm{G}}
$$

140 where

$$
\pi_{\mathrm{J}}=p_{\mathrm{J}}-\left(\frac{\partial F_{\text {int }}}{\partial \varphi_{\mathrm{J}}}\right)_{S_{\mathrm{L}}, \varphi_{\mathrm{K} \neq \mathrm{J}}}
$$

142 as follows: 
(i) $\pi_{\mathrm{J}} d \varphi_{\mathrm{J}}$ is the infinitesimal deformation work given to the solid matrix through the partial pore deformation $d \varphi_{\mathrm{J}}$.

(ii) $\pi_{\mathrm{J}}$ represents, at the macroscopic scale, the modeling of the actual normal stress exerted to the solid matrix. Therefore $\pi_{\mathrm{J}}$ can be coined as an effective pore pressure.

(iii) $p_{\mathrm{J}}-\pi_{\mathrm{J}}=\frac{\partial F_{\mathrm{int}}}{\partial \varphi_{\mathrm{J}}}$ is due to the surface tension sustained by the solid-fluid interface and can be compared with the Young-Lapace equation ${ }^{1}$.

From the balance energy (5) the state laws read

$$
\sigma_{i j}=\left(\frac{\partial F_{\mathrm{sol}}}{\partial \epsilon_{i j}}\right)_{S_{\mathrm{L}}, \varphi_{\mathrm{J}}} \quad \pi_{\mathrm{J}}=\left(\frac{\partial F_{\mathrm{sol}}}{\partial \varphi_{\mathrm{J}}}\right)_{S_{\mathrm{L}}, \epsilon_{i j}, \varphi_{\mathrm{K} \neq \mathrm{J}}}
$$

At constant saturation degree $S_{\mathrm{L}}$, the linearization of the state laws $(7)$ can provide a first approach of the constitutive equations of unsaturated soils. However the coefficients involved in the linearization process must depend on $S_{\mathrm{L}}$. As a general rule the variable $S_{\mathrm{L}}$ appearing in the arguments of $F_{\text {sol }}$ should be considered as a coupling term only. As a consequence expression for $F_{\text {sol }}$ should involve only small terms as strains and partial pore deformations: $\epsilon_{i j}, \varphi_{\mathrm{J}}$.

On the other way, a general requirement for $F_{\text {sol }}$ can be expressed as follows. Along any loading path characterized by $\pi_{\mathrm{L}}=\pi_{\mathrm{G}}$ the solid wall is subjected to a uniform pore pressure. In that case, according to Eq. (5), we could expect an expression for $F_{\text {sol }}$ which is independent of $S_{\mathrm{L}}$ as long as $\pi_{\mathrm{L}}=\pi_{\mathrm{G}}$. In other

\footnotetext{
${ }^{1}$ In case of a spherical pore of radius $r$, it is easy to show that $\frac{\partial F_{\text {int }}}{\partial \varphi_{\mathrm{J}}}=\frac{2 \gamma}{r}$ where $\gamma$ is the surface tension
} 
words the derivative $\frac{\partial F_{\text {sol }}}{\partial S_{\mathrm{L}}}$ should vanish for any value $\pi_{\mathrm{L}}=\pi_{\mathrm{G}}$ :

$$
\left(\frac{\partial F_{\mathrm{sol}}}{\partial S_{\mathrm{L}}}\right)_{\epsilon_{i j}, \varphi_{\mathrm{K}}}=0 \quad \forall \pi_{\mathrm{L}}=\pi_{\mathrm{G}}
$$

162

$$
F_{\text {int }}\left(\varphi_{\mathrm{L}}+\lambda \phi_{0} S_{\mathrm{L}}, \varphi_{\mathrm{G}}+\lambda \phi_{0} S_{\mathrm{G}}, S_{\mathrm{L}}\right)=\left(1+\frac{2}{3} \lambda\right) F_{\mathrm{int}}\left(\varphi_{\mathrm{L}}, \varphi_{\mathrm{G}}, S_{\mathrm{L}}\right) \quad \forall \lambda \ll 1
$$

173 Statement (10) expresses that the interface energy change, at constant satura174 tion, is only due to the change of the surface areas of pores in the process of

${ }_{177}(1+\lambda)$ (i.e the volume of pore $\phi_{0} S_{\mathrm{J}}$ would increase by $\left.\lambda \phi_{0} S_{\mathrm{J}}\right)$ and an increase of 178 surface by a factor $\left(1+\frac{2}{3} \lambda\right)$. Property (10) implicitly assumes that the surface

Since the previous approach has postulated that, at the macroscopic scale, this energy only depends on the 3 thermodynamical variables, $\left(\varphi_{\mathrm{L}}, \varphi_{\mathrm{G}}, S_{\mathrm{L}}\right)$, such an expression must be consistent with expression (9) for any deformation process. To go further we are going to make some reasonable assumptions for interface energy. Surface tensions are assumed constant or only temperature dependent. Accordingly since interface energy is spread over surfaces we can assume the following property regarding the dependence of $F_{\text {int }}$ upon the partial pore deformations:

deformation. Indeed from the current state any virtual (small) homogeneous dilation of coefficient $\left(1+\frac{1}{3} \lambda\right)$ would cause an increase of volume by a factor 
181 to $\lambda$ entails

$$
\phi_{0} S_{\mathrm{L}}\left(\frac{\partial F_{\text {int }}}{\partial \varphi_{\mathrm{L}}}\right)_{\epsilon_{i j}, \varphi_{\mathrm{G}}}+\phi_{0} S_{\mathrm{G}}\left(\frac{\partial F_{\text {int }}}{\partial \varphi_{\mathrm{G}}}\right)_{\epsilon_{i j}, \varphi_{\mathrm{L}}}=\frac{2}{3} F_{\mathrm{int}}
$$

182

Finally linearizing $F_{\text {int }}$ with respect to the partial pore deformations $\varphi_{\mathrm{J}}$ gives

$$
F_{\text {int }}=\frac{2}{3} U_{\mathrm{L}}\left(S_{\mathrm{L}}\right) \varphi_{\mathrm{L}}+\frac{2}{3} U_{\mathrm{G}}\left(S_{\mathrm{L}}\right) \varphi_{\mathrm{G}}+\phi_{0} U\left(S_{\mathrm{L}}\right)
$$

where $U$ is the interface energy per unit of porous space prior to any deformation process. Combining (11) and (12) shows that $U$ is expressed as

$$
U=S_{\mathrm{L}} U_{\mathrm{L}}+S_{\mathrm{G}} U_{\mathrm{G}}
$$

185 where $U_{\mathrm{L}}$ and $U_{\mathrm{G}}$ are two interface energies associated to the liquid and gas

186 phases. From (6) we have

$$
\pi_{\mathrm{J}}=p_{\mathrm{J}}-\frac{2}{3} U_{\mathrm{J}}
$$

${ }_{187}$ With the help of the equations derived above the interface energy balance writes

$$
d F_{\text {int }}=\left(p_{\mathrm{L}}-\pi_{\mathrm{L}}\right) d \varphi_{\mathrm{L}}+\left(p_{\mathrm{G}}-\pi_{\mathrm{G}}\right) d \varphi_{\mathrm{G}}-\left(\phi_{0}\left(p_{\mathrm{G}}-p_{\mathrm{L}}\right)+\frac{\partial F_{\mathrm{sol}}}{\partial S_{\mathrm{L}}}\right) d S_{\mathrm{L}}
$$

${ }_{188}$ Neglecting $\frac{\partial F_{\text {sol }}}{\partial S_{\mathrm{L}}}$ compared to $\frac{\partial F_{\text {int }}}{\partial S_{\mathrm{L}}}$, the state laws of interface now read at the 189 first order

$$
p_{\mathrm{G}}-p_{\mathrm{L}}=-\frac{d U}{d S_{\mathrm{L}}} ; \quad p_{\mathrm{L}}-\pi_{\mathrm{L}}=\frac{2}{3} U_{\mathrm{L}} ; \quad p_{\mathrm{G}}-\pi_{\mathrm{G}}=\frac{2}{3} U_{\mathrm{G}}
$$

These 3 state laws can be compared, in some way, with a kind of macroscopic

${ }_{191}$ Young-Laplace law. The first law (16a) is the well known capillary or retention 
cure. The two other laws $(16 \mathrm{~b}, 16 \mathrm{c})$ are unusual and difficult to apply because it

193 is not possible to measure energies $U_{\mathrm{J}}$ separately. One possible way to overcome

194 this difficulty comes from an exploitation of the microscopic definition of the interface energy (9) which can be written, in the undeformed state and using

196 the Young equation, as

$$
\phi_{0} U=\gamma_{\mathrm{SL}}\left(\omega_{\mathrm{SL}}-\frac{\omega_{\mathrm{GL}}}{\cos \theta}\right)+\gamma_{\mathrm{SG}}\left(\omega_{\mathrm{SG}}+\frac{\omega_{\mathrm{GL}}}{\cos \theta}\right)
$$

${ }_{197}$ where $\theta$ is the contact angle of the liquid assumed as the wetting phase. Then 198 we assume that each term of the rhs of Eq. (17) can be identified to that of the rhs of Eq. (13). Using the property that the sum $\omega_{\mathrm{SL}}+\omega_{\mathrm{SG}}$ is the total

200 surface of the solid wall (and therefore is constant), we can derive an expression 201 of $S_{\mathrm{J}} U_{\mathrm{J}}$ in the form

$$
\begin{aligned}
S_{\mathrm{L}} U_{\mathrm{L}} & =U(1)-\frac{\gamma_{\mathrm{SL}}}{\gamma_{\mathrm{SG}}-\gamma_{\mathrm{SL}}}\left(U\left(S_{\mathrm{L}}\right)-U(1)\right) \\
S_{\mathrm{G}} U_{\mathrm{G}} & =\frac{\gamma_{\mathrm{SG}}}{\gamma_{\mathrm{SG}}-\gamma_{\mathrm{SL}}}\left(U\left(S_{\mathrm{L}}\right)-U(1)\right)
\end{aligned}
$$

202 where $U(1)$ can be set to 0 by considering the saturated state as a reference state. ${ }_{203}$ Since liquid is the wetting phase the fraction $\frac{\gamma_{\mathrm{SL}}}{\gamma_{\mathrm{SG}}-\gamma_{\mathrm{SL}}}$ is a positive number that 204 we will denote by $a$, in the following, so that

$$
S_{\mathrm{L}} U_{\mathrm{L}}=-a U\left(S_{\mathrm{L}}\right) ; \quad S_{\mathrm{G}} U_{\mathrm{G}}=(1+a) U\left(S_{\mathrm{L}}\right)
$$
We have to point out that the identification (20) relies on the assumption, albeit 206 natural, that the rhs of (17) and (13) can be identified term by term. Moreover ${ }_{207}$ because the surface tensions $\gamma_{\mathrm{SJ}}$ are generally not known, the coefficient $a$ should be calibrated directly at the macroscopic scale. 
To derive this model, we will assume that along any loading path defined by a ${ }_{211}$ constant saturation degree, $d S_{\mathrm{L}}=0$, and constant effective pressures, $d \pi_{\mathrm{J}}=0$, ${ }_{212}$ the partial pore deformation increment, $d \varphi_{\mathrm{J}}$ is a saturation dependent fraction 213 of the total pore deformation:

$$
\left(d \varphi_{\mathrm{L}}\right)_{S_{\mathrm{L}}, \pi_{\mathrm{J}}}=\chi d \varphi ; \quad\left(d \varphi_{\mathrm{G}}\right)_{S_{\mathrm{L}}, \pi_{\mathrm{J}}}=(1-\chi) d \varphi
$$

214 223 states:

$$
\delta\left(0, \pi_{\mathrm{L}}, \pi_{\mathrm{G}}\right)=\delta\left(1, \pi_{\mathrm{L}}, \pi_{\mathrm{G}}\right)=0
$$

224 Incorporating expression (22) for $\varphi_{\mathrm{J}}$ in (5) gives

$$
\left(d F_{\mathrm{sol}}\right)_{S_{\mathrm{L}}}=\sigma_{i j} d \epsilon_{i j}+\pi d \varphi+\Delta d \delta
$$




$$
\begin{aligned}
\pi & =\chi \pi_{\mathrm{L}}+(1-\chi) \pi_{\mathrm{G}} \\
\Delta & =\pi_{\mathrm{L}}-\pi_{\mathrm{G}}
\end{aligned}
$$

${ }_{226}$ Defining the Legendre-Fenchel transform $F_{\mathrm{sol}}^{*}=F_{\mathrm{sol}}-\sigma_{i j} \epsilon_{i j}-\pi \varphi-\Delta \delta$ entails

$$
\left(d F_{\mathrm{sol}}^{*}\right)_{S_{\mathrm{L}}}=-\epsilon_{i j} d \sigma_{i j}-\varphi d \pi-\delta d \Delta
$$

${ }_{227}$ Since $\delta$ only depends on $\left(S_{\mathrm{L}}, \pi, \Delta\right)$, the integration of the state equation

$$
\delta\left(S_{\mathrm{L}}, \pi, \Delta\right)=-\left(\frac{\partial F_{\mathrm{sol}}^{*}}{\partial \Delta}\right)_{S_{\mathrm{L}}, \sigma_{i j}, \pi}
$$

228 shows that $F_{\text {sol }}^{*}$ can be split as follows

$$
F_{\mathrm{sol}}^{*}=F_{\mathrm{sol}}^{* 1}\left(\sigma_{i j}, \pi, S_{\mathrm{L}}\right)-\int_{0}^{\Delta} \delta\left(S_{\mathrm{L}}, \pi, u\right) d u
$$

${ }_{232} F_{\text {sol }}^{* 1}$ only depends on $\left(\sigma_{i j}, \pi\right)$ with

$$
d F_{\mathrm{sol}}^{* 1}=-\epsilon_{i j} d \sigma_{i j}-\varphi^{*} d \pi
$$

233 where we defined

$$
\varphi^{*}=\varphi-\int_{0}^{\Delta} \frac{\partial \delta}{\partial \pi}\left(S_{\mathrm{L}}, \pi, u\right) d u
$$

${ }_{234}$ Expression (31) for $\varphi^{*}$ can be transformed by using the Maxwell symmetry 235 relation derived from $(27)$

$$
\begin{aligned}
\left(\frac{\partial \delta}{\partial \pi}\right)_{S_{\mathrm{L}}, \Delta} & =\left(\frac{\partial \varphi}{\partial \Delta}\right)_{S_{\mathrm{L}}, \sigma_{i j}, \pi} \\
{ }^{2} \text { where we can notice that }\left(\frac{\partial F_{\mathrm{sol}}}{\partial S_{\mathrm{L}}}\right)_{\epsilon_{i j}, \varphi, \delta} & =\left(\frac{\partial F_{\mathrm{sol}}^{*}}{\partial S_{\mathrm{L}}}\right)_{\sigma_{i j}, \pi, \Delta}
\end{aligned}
$$



We obtained

$$
\varphi^{*}=\left.\varphi\right|_{\Delta=0}
$$

Hence $\varphi^{*}$ turns out to be the total pore deformation that would have resulted from $\Delta=0$, i.e. $\pi_{\mathrm{L}}=\pi_{\mathrm{G}}$, while keeping the other variables as constant.

Therefore expression $F_{\text {sol }}^{* 1}\left(\sigma_{i j}, \pi\right)$ which is independent of $S_{\mathrm{L}}$, must have the same expression as that found under saturated state. The state equations

$$
\epsilon_{i j}=-\left(\frac{\partial F_{\mathrm{sol}}^{* 1}}{\partial \sigma_{i j}}\right)_{\pi} \quad \varphi^{*}=-\left(\frac{\partial F_{\mathrm{sol}}^{* 1}}{\partial \pi}\right)_{\sigma_{i j}}
$$

show that $\pi$ play the role of an equivalent pore pressure in the sense that it would be the pressure to apply to the liquid phase of the porous material under saturated state, to get the same strains as those obtained under unsaturated state, at the given stress state $\sigma_{i j}$. Note however that the total pore deformation that would be obtained under saturated state is $\varphi^{*}$ and not that of the unsaturated state, $\varphi$.

Assuming that, at the microscopic scale, the solid matrix is isotropic and behaves elastically, with a compressibility constant $k_{s}$, an incremental loading defined, at constant saturation degree, by $d \sigma=-d \pi_{\mathrm{L}}=-d \pi_{\mathrm{G}}=-d \pi$, will load the solid grains by a uniform increment of pressure leading to a response given by $d \epsilon_{i i}=d \varphi / \phi_{0}=d \varphi_{\mathrm{J}} /\left(\phi_{0} S_{\mathrm{J}}\right)=-d \pi / k_{s}$. Incorporating these last equations 52 into the general equation (22) leads to

$$
\left(\frac{\partial \delta}{\partial \pi}\right)_{S_{\mathrm{L}}, \Delta}=\left(\frac{\partial \varphi}{\partial \Delta}\right)_{S_{\mathrm{L}}, \sigma_{i j}, \pi}=\frac{\phi_{0}\left(\chi-S_{\mathrm{L}}\right)}{k_{s}}
$$

253 showing that $\delta$ and $\varphi$ vary linearly with $\pi$ and $\Delta$, respectively. Accordingly we 
obtain for $\varphi$

$$
\varphi=\varphi^{*}+\frac{\phi_{0}\left(\chi-S_{\mathrm{L}}\right)}{k_{s}} \Delta
$$

255 showing that $\varphi^{*}=\varphi$ in the iso-deformation assumption, $\chi=S_{\mathrm{L}}$.

Furthermore the $\delta-\Delta$ relationship is expected to involve the elastic shear ${ }_{257}$ property of the solid matrix. If we assume the linearity of such behavior we end 258 up with

$$
\delta=\frac{\phi_{0}\left(\chi-S_{\mathrm{L}}\right)}{k_{s}} \pi+\frac{\Delta}{g\left(S_{\mathrm{L}}\right)}
$$

259 where $g\left(S_{\mathrm{L}}\right)$ stands for an elastic modulus characterizing the elastic shear prop260 erty of the solid matrix.

${ }_{261}$ When the solid grains are incompressible, $k_{s}$ can be set to $\infty$ in the previous 262 equations. The volumetric deformation of the soil is then equal to the total pore 263 deformation, $\epsilon_{v}=\varphi=\varphi^{*}$, and the balance (30) turns into

$$
d F_{\mathrm{sol}}^{* 1}=-\epsilon_{v} d(\sigma+\pi)-\epsilon_{q} d q
$$

${ }_{264}$ where $q$ and $\epsilon_{q}$ are the deviatoric stress and strain respectively. From (38)

$$
\epsilon_{v}=-\left(\frac{\partial F_{\mathrm{sol}}^{* 1}}{\partial(\sigma+\pi)}\right)_{q}
$$

\section{${ }_{265} 4$ Elastic behavior}

${ }_{266}$ Under isotropic loading, the mechanical response of saturated soils is well de${ }_{267}$ scribed by a constant compressibility coefficient, $\kappa$, in the form

$$
d e=-\kappa \frac{d(\sigma+p)}{\sigma+p}
$$


273 a comparison of (40) and (39) implies the general constitutive relationship

$$
d e=-\kappa \frac{d(\sigma+\pi)}{\sigma+\pi}
$$

${ }_{274}$ which should be valid for unsaturated situations and along any loading paths 275 and with the same constant compressibility coefficient as that involved in (40).

${ }_{277} \pi$, has to be computed from (25). This can be done from the water retention

${ }_{286} \chi=S_{\mathrm{L}}$. The reported measured points are found to be accurately lined up with ${ }_{287}$ a slope $\kappa=0.0978$, namely very close to that found in the previous experiment.

However to be able to apply the previous relation, the equivalent pore pressure, curve of the material. This curve has been measured, at 20 and $80{ }^{\circ} \mathrm{C}$, on the same FoCa clay using the saturated salt solution technique upon imbibition path [14]. This curve is shown in the figure (2a). During these tests no stress was applied thereby, neglecting the atmospheric pressure, $\sigma=0$. In these conditions Eq. (41) turns into

$$
d e=-\kappa \frac{d \pi}{\pi}
$$

283 The free swelling of these samples was measured and reported in figure (2b) in terms of $\pi$ calculated with the help of the measured retention curves, the gas pressure being neglecting. We assume here the iso-deformation of pores, i.e. (a) 


\section{$5 \quad$ Plastic Modeling}

293 inequality in the form [11]

$$
\sigma_{i j} d \epsilon_{i j}+\pi_{\mathrm{L}} d \varphi_{\mathrm{L}}+\pi_{\mathrm{G}} d \varphi_{\mathrm{G}}-\left(d F_{\mathrm{sol}}\right)_{S_{\mathrm{L}}} \geq 0
$$
ing 302 and plastic part of the deformation variables, respectively, we write

$$
F_{\text {sol }}=W\left(\epsilon_{i j}^{e}, \varphi_{\mathrm{L}}^{e}, \varphi_{\mathrm{G}}^{e}, S_{\mathrm{L}}\right)+Z\left(S_{\mathrm{L}}, \alpha\right)
$$
allows to write the Clausius-Duhem inequality as

We will assume in the following that irreversibility only affects the mechanical behavior. Hysteresis of the retention curve will not be addressed here. As a consequence, in non reversible transformation, the two first laws of thermodynamics applied to the system composed of the solid matrix, gives the Clausius-Duhem

where now the free energy of the solid matrix, $F_{\text {sol }}$, must be argumented by the elastic part of the deformation variables and by hardening variables. Following [13] this energy is split into two parts: (i) the elastic energy, $W$, stored in the solid matrix which is the energy recoverable by a reversible mechanical process and (ii) the locked energy, $Z$, which is the additional (not recoverable) part of the elastic energy locked when irreversible mechanical processes have taken place. For the sake of simplicity, the locked energy is assumed to depend on a unique hardening variable, $\alpha$. Denoting with superscripts $e$ and $p$ the elastic

so3 The state equations (7) being always valid provided that each deformation vari${ }_{04}$ able be replaced by its elastic part, the use of expression (44) for $F_{\text {sol }}$ in (43)

$$
\sigma_{i j} d \epsilon_{i j}^{p}+\pi_{\mathrm{L}} d \varphi_{\mathrm{L}}^{p}+\pi_{\mathrm{G}} d \varphi_{\mathrm{G}}^{p}+\beta d \alpha \geq 0
$$


where $\beta$ is defined by

$$
\beta=-\left(\frac{\partial Z}{\partial \alpha}\right)_{S_{\mathrm{L}}}
$$

${ }_{307}$ The variable $\beta$ is the hardening force as energy conjugate to the hardening ${ }_{308}$ variable $\alpha$. It will be associated to the current limit of the elastic domain 309 defined by

$$
f\left(\sigma, \pi_{\mathrm{L}}, \pi_{\mathrm{G}}, \beta\right) \leq 0
$$

310 Following the work of Coussy [13], to go further towards an effective stress, we ${ }_{311}$ will assume that part of the flow rule is given by

$$
\left(d \varphi_{\mathrm{L}}^{p}\right)_{S_{\mathrm{L}}}=\chi^{p} d \varphi^{p} ; \quad\left(d \varphi_{\mathrm{G}}^{p}\right)_{S_{\mathrm{L}}}=\left(1-\chi^{p}\right) d \varphi^{p}
$$

312 where $\chi^{p}$ is a weighting factor ranging from 0 to 1 . This factor is, a priori, ${ }_{313}$ different from $\chi$ which was introduced previously to describe the elastic response. 314 Similarly to what was done for $\chi$, we will assume that this factor depends 315 on the saturation degree: $\chi^{p}\left(S_{\mathrm{L}}\right)$. The plastic incompressibility of the solid 316 grains is now introduced leading to $\epsilon_{v}^{p}=\varphi^{p}$. From the plastic incompressibility 317 assumption and (48) the dissipation (45) turns into

$$
\sigma^{\mathrm{B}} d \epsilon_{v}^{p}+q d \epsilon_{q}^{p}+\beta d \alpha \geq 0
$$

318 where $\sigma^{\mathrm{B}}$ is a Bishop-like stress defined by

$$
\sigma^{\mathrm{B}}=\sigma+\chi^{p} \pi_{\mathrm{L}}+\left(1-\chi^{p}\right) \pi_{\mathrm{G}}
$$

319 According to (50) and (49) the current elastic domain can be defined by

$$
f\left(\sigma^{\mathrm{B}}, q, \beta\right) \leq 0
$$


The flow rule is then expressed in the form

$$
d \epsilon_{v}^{p}=d \lambda\left(\frac{\partial f}{\partial \sigma^{\mathrm{B}}}\right)_{q, \beta} \quad d \epsilon_{q}^{p}=d \lambda\left(\frac{\partial f}{\partial q}\right)_{\sigma^{\mathrm{B}}, \beta}
$$

321 One of the simplest plastic model used for saturated clay is the Modified Cam-

322 Clay model:

$$
f_{\text {Cam }}\left(\sigma, q, p_{0}\right)=\sigma\left(\sigma+p_{0}\right)+q^{2} / M^{2}
$$

${ }_{323}$ where $p_{0}$ is the preconsolidation pressure at the saturated state which is gov-

324 erned by the plastic void ratio:

$$
p_{0}=p_{r} \exp \left(-\frac{e^{p}}{\lambda(0)-\kappa}\right)
$$

325 where $\lambda(0)$ is the slope of the saturated virgin consolidation line while $\kappa$ is the 326 slope of the unloading/reloading line as introduced in section 4. A simple exten327 sion of the yield function (53) to the unsaturated state can then be formulated 328 as

$$
f=f_{\text {Cam }}\left(\sigma^{\mathrm{B}}, q, p_{0}\right)
$$

329 where the preconsolidation pressure $p_{0}$ should be extended to unsaturated situations. Following the work of Coussy [13], we set

$$
p_{0}=p_{r} h\left(e^{p}, S_{\mathrm{L}}\right)
$$

331 where $h$ should satisfy $h\left(e^{p}, 1\right)=\exp \left(-\frac{e^{p}}{\lambda(0)-\kappa}\right)$.

332 In the following we will assume that

$$
h\left(e^{p}, S_{\mathrm{L}}\right)=h_{m}\left(e^{p}\right) h_{s}\left(S_{\mathrm{L}}\right)
$$

${ }_{333}$ where $h_{m}=\exp \left(-\frac{e^{p}}{\lambda(0)-\kappa}\right)$ expresses the mechanical hardening due to irre334 versible deformations and $h_{s}$ represents a saturation-induced hardening (which 


$$
\tau=C-\left(\sigma_{n}+\chi^{p} \pi_{\mathrm{L}}+\left(1-\chi^{p}\right) \pi_{\mathrm{G}}\right) \tan \psi
$$

${ }^{41}$ where $C$ is the cohesion and $\psi$ is the friction angle which is assumed constant, consistently with the Cam-Clay model: $\sin \psi=3 M /(6+M)$. Alonso et al [3] have shown that the coefficient $\chi^{p}$ to consider in the shear strength of unsaturated soils is actually smaller than the saturation degree. They proposed a generic formula of the form

$$
\chi^{p}=\left\langle\frac{S_{\mathrm{L}}-S_{\mathrm{L}}^{m}}{1-S_{\mathrm{L}}^{m}}\right\rangle
$$

346 where $\langle x\rangle=(x+|x|) / 2$ is the positive part operator. Alonso et al related ${ }_{347}$ this coefficient (called effective degree of saturation in their paper) to the freely 348 available water filling the macroporosity of the soil. Here we will rather use 349 this formula as a parametric form of the coefficient $\chi^{p}$. From (20) and (14), a 350 development of Eq. (58) in terms of the capillary pressure, $p_{c}=p_{\mathrm{G}}-p_{\mathrm{L}}$, and 351 interface energy, $U$, gives

$$
\tau=C-\left(\sigma_{n}+p_{\mathrm{G}}-\chi^{p} p_{c}-\frac{2}{3}\left(-\frac{\chi^{p}}{S_{\mathrm{L}}} a+\frac{1-\chi^{p}}{1-S_{\mathrm{L}}}(1+a)\right) U\right) \tan \psi
$$


352 Numerous experiments have been performed on shear strength of unsaturated 353 soils. We present in the figure (3) those performed on Guadalix de la Sierra red 354 clay [16] for which $C=0$ and $\psi=33^{\circ}$. In the same figure we have plotted the 355 model as predicted by Eq. (60) under different hypotheses: $\chi^{p}=S_{\mathrm{L}}$ or $\chi^{p}$ given 356 by Eq. (59). The model of Brooks and Corey [8] was used to fit the retention 357 curve with an air entry pressure of $30 \mathrm{kPa}$ and an exponent equal to 3.

\section{${ }_{358} \quad 5.2$ Isotropic stress path at constant capillary pressure}

359 The compression index of a normally consolidated saturated soil is defined by

$$
d e=-\lambda(0) \frac{d(\sigma+p)}{\sigma+p}
$$

360 For an unsaturated soil at constant capillary pressure, an isotropic plastic load-

361 ing on the virgin compression line, i.e $-\sigma^{\mathrm{B}}=p_{0}$, results in

$$
d e^{p}=-(\lambda(0)-\kappa) \frac{d \sigma^{\mathrm{B}}}{\sigma^{\mathrm{B}}}
$$

362 Assuming that $\chi=\chi^{p}$ entails

$$
d e=-\lambda(0) \frac{d \sigma^{\mathrm{B}}}{\sigma^{\mathrm{B}}}
$$

${ }_{363}$ which is the simplest extension of (61) for unsaturated situations. We assume 364 moreover, hereafter, that $\chi=\chi^{p}=S_{\mathrm{L}}$. We have applied this simple model to 365 a silty clay $[25,26,35]$. The figure (4b) reports the variation of the void ratio 366 during a compression oedometric test from 1 to $256 \mathrm{kPa}$ obtained at different 367 capillary pressures: $p_{c}=0,20,40,80,160 \mathrm{kPa}$. The figure (4a) shows the predic368 tion obtained by the model. The elastic limit, given by $-\sigma=\pi+p_{0}$, has been 
identified to $18,22,24,28,50 \mathrm{kPa}$ respectively. From these results we have identified the following parameters associated to the saturated state: $\lambda(0)=0.037$, $\kappa=0,004$ and $p_{r}=18 \mathrm{kPa}$. To assess the value of the interface energy, $U$, we use a retention curve fitted by using the model of Brooks and Corey with the parameters associated to the silty clay (air entry pressure $p_{e}=10 \mathrm{kPa}$ and exponent $\alpha=2.5)$.

\subsection{Imbibition drainage paths}

The same silty clay as that used in the previous section was loaded at different isotropic compression stresses: $-\sigma=8,16,32,64,128,256 \mathrm{kPa}$. After each compression test, the capillary pressure is increased from 0 to $160 \mathrm{kPa}$ then the specimen is unloaded to its initial compression, i.e $1 \mathrm{kPa}$. The same parameters as those defined in the last section are used. Figure (5a) shows the void ratio variations during the different loading paths. The vertical lines correspond to the capillary pressure load. The corresponding evolution of the void ratio is represented in the figure (6a). The experimental results are represented in the figures (5b) and (6b).

Let's consider now a soil sample initially and normally saturated. The initial capillary pressure is equal to zero. Let's submit the specimen to increasing capillary pressure from 1 to $256 \mathrm{kPa}$. The capillary pressure is then decreased to $1 \mathrm{kPa}$. We use the following parameters $\lambda(0)=0.19, \kappa=0.031, p_{r}=10 \mathrm{kPa}$, and an initial stress $-\sigma_{0}=1.510^{-6} \mathrm{kPa}$. The parameters of the retention curve (Brook and Corey) are given by $p_{e}=1.8 \mathrm{MPa}$ and $\alpha=1$. These parameters 

corrrespond to a white clay used in [17]. The experiments are shown in the ${ }_{392}$ figure (7b) and can be compared to the predictions shown in the figure (7a). In 393 the experiment the sample is not mechanically loaded. So we found the initial ${ }_{394}$ stress in order to fit one point of the curve. We found the very small value $-\sigma_{0}=$ ${ }_{395} 1.510^{-6} \mathrm{kPa}$. During the loading path from 1 to $1.8 \mathrm{kPa}$ the capillary pressure is 396 lower than the air entry pressure and the soil is actually saturated. The slope of the curve is the compression index. For capillary pressure greater than $1.8 \mathrm{kPa}$

398 the sample behaves elastically because we assumed that the saturation-induced 399 hardening $h_{s}\left(S_{\mathrm{L}}\right)>1+\frac{\chi^{p} p_{c}-p_{e}}{p_{r} h_{m}\left(e^{p}\right)}$, resulting in a strictly negative yield function: $400 \quad f<0$.

\section{${ }_{401} \quad 6$ Conclusion}

${ }_{402}$ The model proposed by Coussy [13] has been extended to account for interface 403 energies in the mechanical behaviour of unsaturated soils. This extension relies 404 on the assumption that the interface energy depends on the partial pore defor405 mations in addition to the saturation degree. As a consequence, effective pore 406 pressures should be considered in the mechanical behaviour in place of the liquid ${ }_{407}$ and gas pressures. These effective pore pressures differ from the pore pressures ${ }_{408}$ by terms involving the interface energy. Following the approach developped in ${ }_{409}$ Coussy et al, a Bishop-like effective stress, expressed in terms of these effective 410 pore pressures, is found to control the mechanical behaviour of unsaturated soils ${ }_{411}$ providing an assumption concerning the partial pore deformations, i.e the de- 
412 formation of the partial volume occupied by the fluids. The Bishop's coefficient,

${ }_{413} \chi$, turns out to be a saturation dependent fraction of the partial pore deforma-

${ }_{414}$ tion and the total pore deformation. Actually two Bishop-like effective stresses,

415 associated to the elastic and plastic behaviour, can be introduced. This results

416 in a model relying on two effective stresses which can be used to extend the

${ }_{417}$ elastic and plastic behaviour of saturated soils to unsaturated conditions. We

418 propose a very simple model based on the extension of the Cam-Clay model.

419 This model is applied to predict the response of a soil sample to compression

${ }_{420}$ stress at constant capillary pressure and to wetting drying paths at constant

${ }_{421}$ stress. These responses are compared with some experimental results reported

422 from the literature.

\section{${ }_{423}$ References}

${ }_{424}$ [1] G.D. Aitchison. Separate roles of site investigation, quantification of soil properties and selection of operational environment in the determination of foundation design on expansive soils. In Proc 3rd Asian Regional Conf. Soil Mech. Found. Engng, volume 3, pages 72-77, Haifa, Israel, 1967.

[2] E.E. Alonso, A. Gens, and A. Josa. A constitutive model for partially saturated soils. Géotechnique, 30:504-430, 1990.

[3] E.E. Alonso, J.M. Prereira, J. Vaunat, and S. Olivella. A microstructurally based effective stress for unsaturated soils. Géotechnique, 60(12):913-925, 2010. 
[4] A. W. Bishop. The principle of effective stress. Teknish Ukeblad, 39:859$863,1959$.

[5] A. W. Bishop and G.E. Blight. Some aspects of effective stress in saturated and partly saturated soils. Géotechnique, 13 (1):177-197, 1963.

[6] G.E. Blight. A study of effective stress for volume change. In G.D. Aitchison, editor, Moisture equilibria and moisture changes in soils beneath covered areas, pages 259-269, Sydney, 1965. Butteworths.

[7] G. Bolzon, B.A. Schrefler, and O.C. Zienkiewicz. Elastoplastic soil constitutive laws generalized to partially saturated states. Géotechnique, 13(3):279$289,1996$.

[8] R.N. Brooks and A.T. Corey. Hydraulic properties of porous media. Colorado State Univ. Hydrol. Paper, 3:270-278, 1964.

[9] J.B. Burland. Some aspects of the mechanical behaviour of partially saturated soils. Moisture Equilibria and Moisture Changes Beneath Covered Areas, pages 270-278, 1965.

[10] X. Chateau and L. Dormieux. Micromechanics of saturated and un saturated porous media. International Journal of Numerical and Analytical Methods in Geomechnaics, 26:831-844, 2002.

[11] O. Coussy. Poromechanics. J. Wiley, 2004. 
[12] O. Coussy. Revisiting the constitutive equations of unsaturated porous solids using a lagrangian saturation concept. Int. J. Numer. Anal. Method, 31:1675-1694, 2007.

[13] O. Coussy, J.M. Pereira, and J. Vaunat. Revisiting the thermodynamics of hardening plasticity for unsaturated soils. Computers and Geotechnics, $37: 207-215,2010$.

[14] P. Dangla, O. Coussy, E. Olchitzky, and C. Imbert. Non linear thermomechanical couplings in unsaturated clay barriers. In W. Ehlers, editor, Theoretical and numerical methods in continuum mechanics of porous materials. Proceedings of IUTAM Symposium. Kluwer Academic Publishers, 2000.

[15] W. Ehlers, T. Graf, and M. Ammann. Deformation and localizaton analysis of partially saturated soil. Comput. Methods Appl. Mech. Engng., 193(2729):2885-2910, 2004.

[16] V. Escario. Formulaciones para la difinicion de la resistancia a esfuerzo cortante de los suelos parcialmente saturados. Ingeniera Civil, 68, 1988.

[17] J. M. Fleureau, S. Kheirbek-Saoud, R. Soemitro, and S. Taibi. Behavior of clayey soils on drying-wetting paths. Canadian Geotechnical Journal, 30:287-296, 1993.

[18] D.G. Fredlund and N.R. Morgenstern. Stress state variables for unsaturated soils. J. of Geot. Eng. Div., ASCE, 103 (GT5):447-466, 1977. 
[19] D. Gallipoli, A. Gens, R. Sharma, and J. Vaunat. An elastoplastic model for unsaturated soil incorporating the effects of suction and degree of saturation on mechanical behaviour. Géotechnique, 53(1):123-135, 2003.

[20] A. Gens and E.E. Alonso. A framework for the behaviour of unsaturated expansive clays. Can. Geotech. J., 29(6):761-773, 1992.

[21] W.G. Gray and B.A. Schrefler. Thermodynamic approach to effective stress in partially saturated porous media. Eur. J. Mech. A/Solids, 20:521-538, 2001.

[22] W.G. Gray and B.A. Schrefler. Analysis of the solid phase stress tensor in multiphase porous media. Int. J. Numer. Anal. Meth. Geomech., 31:541$581,2007$.

[23] J.E.B. Jennings and J.B. Burland. Limitations to the use of effective stresses in partly saturated soils. Géotechnique, 12:125-144, 1964.

[24] Y. Khogo, M. Nakano, and T. Miyazaki. Theoretical aspects of constitutive modelling for unsaturated soils. Soils Found., 33(4):49-63, 1993.

[25] J. Leclercq and J.C. Verbrugge. Considérations relatives à la mécanique des sols non saturés et à son intérêt en agronomie. Bulletin de Recherches Agronomiques, 19:237-267, 1984.

[26] J. Leclercq and J.C. Verbrugge. Propriétés géomécaniques des sols non saturés. In Colloque International "Le travail du sol", pages 1-15, Gembloux le 29/11/1985, Faculté Universitaire des sciences agronomiques, 1985. 
[27] R.W. Lewis and B.A. Schrefler. The finite element method in static and dynamic deformation and consolidation of porous media. John Wiley \& Sons, 1998.

[28] B. Loret and N. Khalili. A three-phase model for unsaturated soils. Int. J. Numer. Anal. Methods Geomech., 24:893-927, 2000.

[29] B. Loret and N. Khalili. An effective stress elastic-plastic model for unsaturated porous media. Mech. Mater., 34:97-116, 2002.

[30] A. Modaressi and N. Abou-Bekr. Constitutive model for unsaturated soils: validation on a silty material. In Proc. Numer. Methods Geomechan., NUMOG $V$, volume 1, pages 91-96, 1994.

[31] M. Nuth and L. Laloui. Effective stress concept in saturated soils: Clarification and validation of a unified framework. Int. J. Numer. Anal. Meth. Geomech., 32:771-801, 2008.

[32] J.M. Pereira, H. Wong, P. Dubujet, and P. Dangla. Adaptation of existing behaviour models to unsaturated states: application to cjs model. International Journal for Numerical and Analytical Methods in Geomechanics, 29:1127-1155, 2005.

[33] R. Santagiuliana and B.A. Schrefler. Enhancing the bolzon-schreflerzienkiewicz constitutive model for partially saturated soil. Trans. Porous Media, 65(1):1-30, 2006. 
514 [34] D. Sheng, S.W. Sloan, and A. Gens. A constitutive model for unsatu515 rated soils: thermomechanical and computational aspects. Comput. Mech., $33(6): 453-465,2004$.

[35] S. Taibi. Comportement mécanique et hydraulique des sols partiellement saturés. PhD thesis, Ecole Central de Paris, 1994.

[36] S.J. Wheeler, R.S. Sharma, and M.S.R. Buisson. Couling of hydraulic hysteresis and stress-strain behaviour in unsaturated soils. Géotechnique, 53(1):41-54, 2003. 


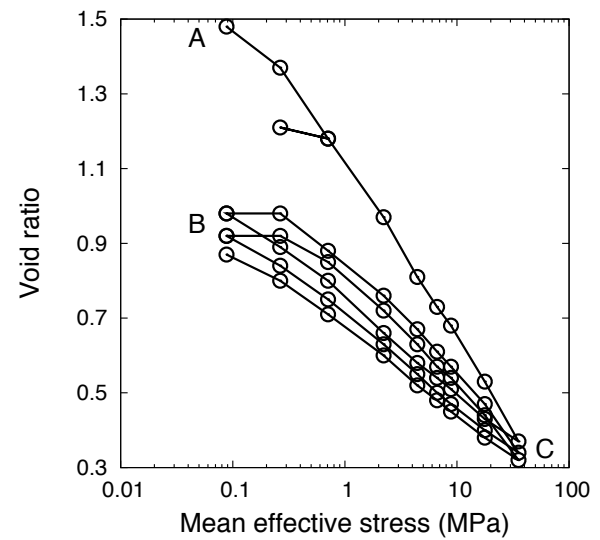

Figure 1: Isotropic compression test performed on saturated FoCa clay [14]. 


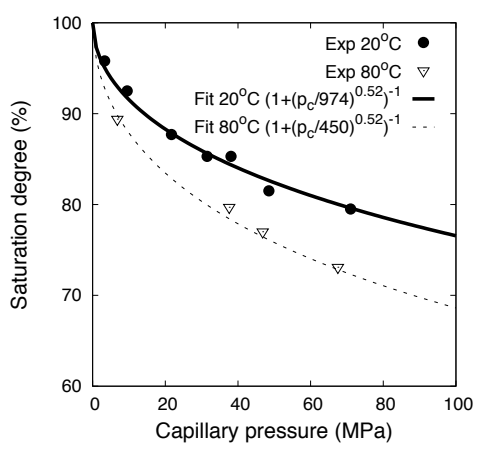

(a)

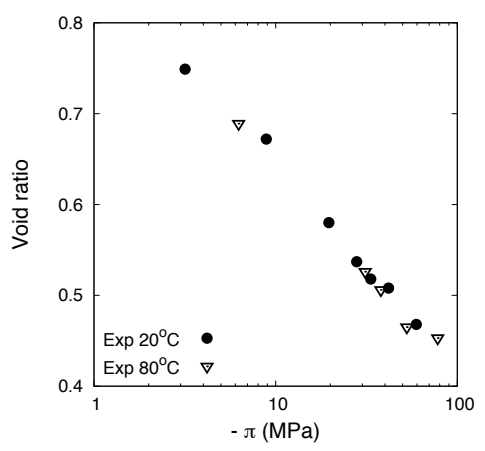

(b)

Figure 2: (a) Retention curve of a FoCa clay obtained by saturated salt solution technique [14]. (b) Void ratio reported against $\log (-\pi)$ in a free swelling during imbibition test. 


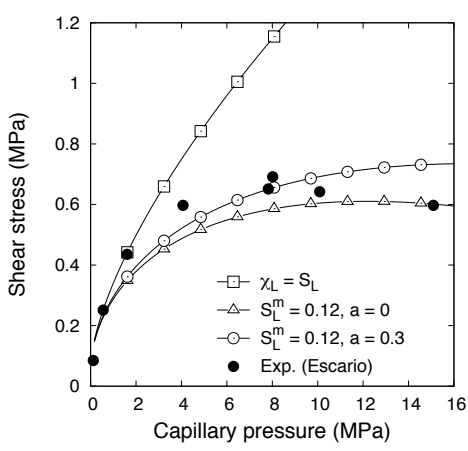

(a)

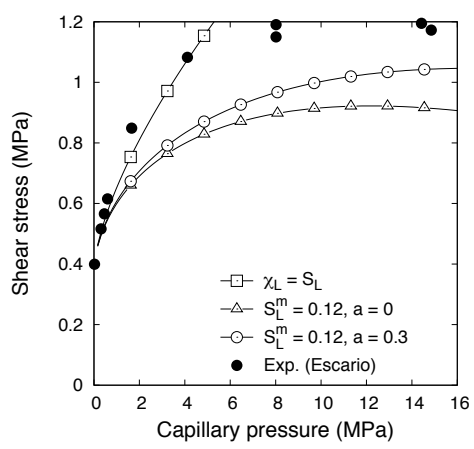

(b)

Figure 3: Shear strength vs capillary pressure obtained at different normal stress: (a) 0.12 MPa (b) 0.6 MPa. The experimental results are reported from Escario et al [16]. 


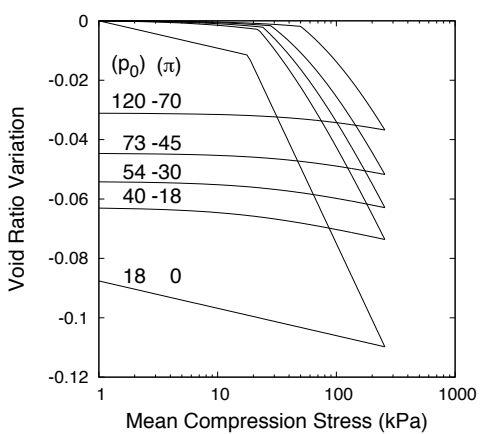

(a)

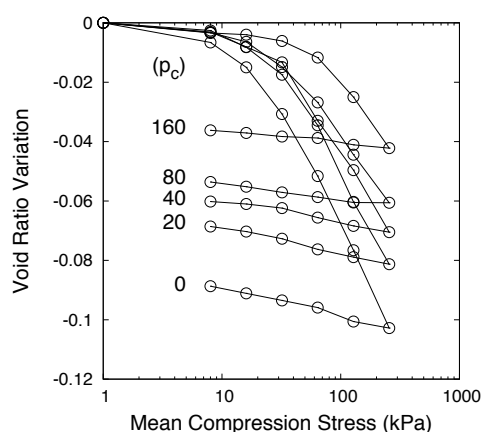

(b)

Figure 4: Isotropic compression curves at constant capillary pressure obtained on a silty clay: (a) Model (b) Experiment reported from [25, 26]. 


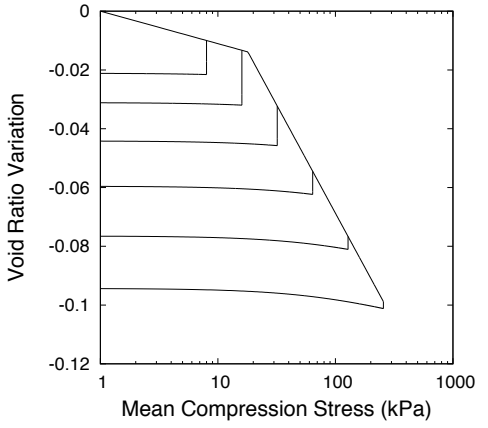

(a)

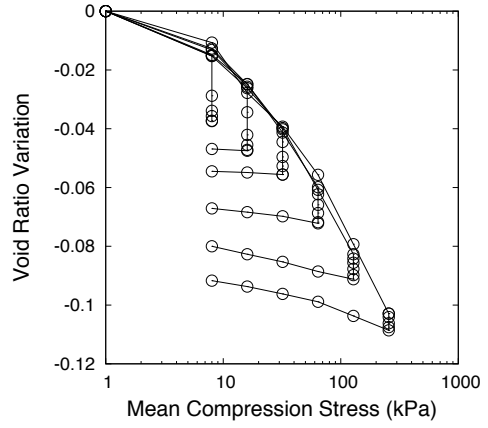

(b)

Figure 5: Compression, drainage and unloading on a silty clay: (a) model (b) experiments $[25,26]$. 


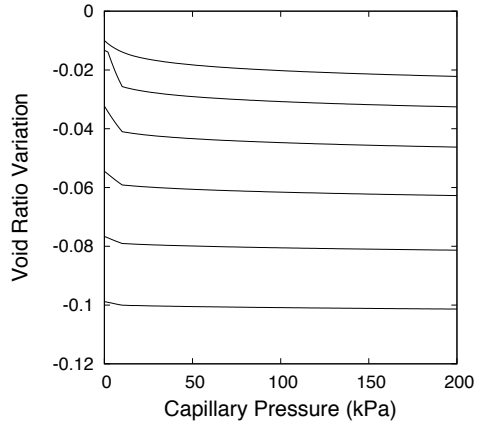

(a)

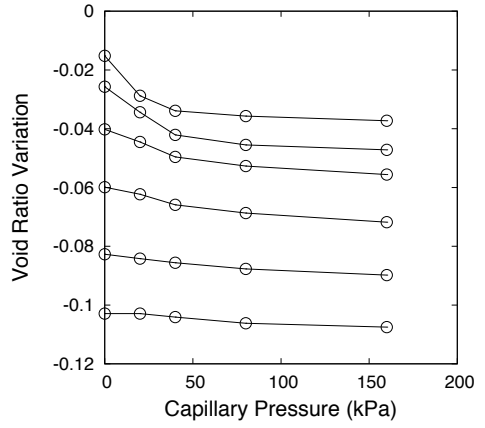

(b)

Figure 6: Drainage phase on a silty clay: (a) model (b) experiments [25, 26]. 


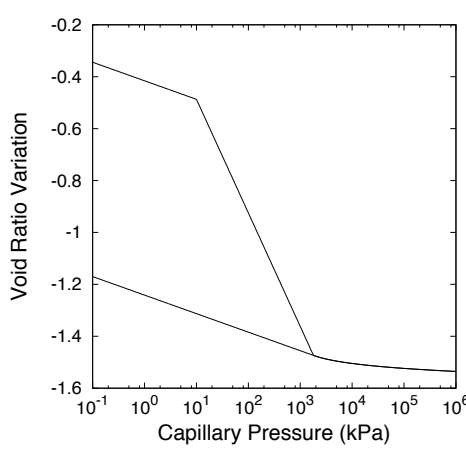

(a)

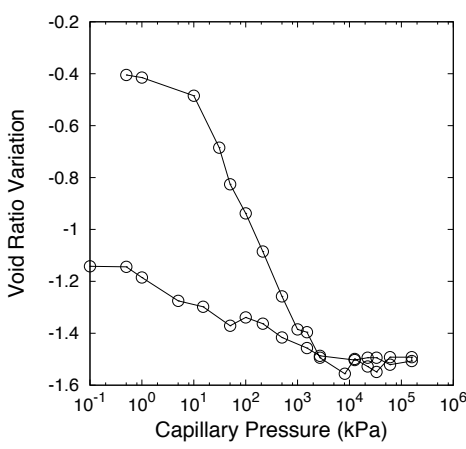

(b)

Figure 7: Drainage on a white clay: (a) model (b) experiments [17]. 